\title{
Model Predictive Control Based Selective Harmonic Mitigation Technique for Multilevel Cascaded H-bridge Converters
}

\author{
Himanshu Aggrawal ${ }^{1}$, Jose I. Leon ${ }^{2}$, Leopoldo G. Franquelo ${ }^{2}$, Samir Kouro ${ }^{3}$, Prashant Garg ${ }^{1}$, and Jose Rodríguez ${ }^{3}$ \\ ${ }^{1}$ Department of Electrical Engineering, \\ Indian Institute of Technology Rajasthan \\ Jodhpur, India, \\ himanshu@ieee.org \\ ${ }^{2}$ Department of Electronics Engineering, \\ University of Seville \\ Seville, Spain, \\ jileon@gte.esi.us.es \\ ${ }^{3}$ Department of Electronics Engineering, \\ Universidad Tecnica Federico Santa Maria \\ Valparaiso, Chile, \\ samir.kouro@ieee.org
}

\begin{abstract}
The selective harmonic elimination (SHE) strategy is specially well suited for high-power applications where the power losses must be kept below strict limits. The SHE technique is based on offline calculations and the generation of a preprogrammed voltage waveforms eliminating some low order harmonics. An evolution of SHE is the selective harmonic mitigation (SHM) technique which is based on pre-programmed waveforms non eliminating the low order harmonics but reducing the distortion below the limits imposed by a grid code. However, the main drawback of these pre-programmed SHE and SHM techniques is a low dynamic performance. In a recent paper, an online SHE technique based on the model predictive control (MPC) has been presented improving the dynamic performance of the conventional SHE method. In this paper, the online version of the SHM technique is introduced. It is based also in the MPC strategy and has been tested in a cascaded multilevel converter obtaining a high performance with very low switching frequency.
\end{abstract}

\section{INTRODUCTION}

The increasing demand of energy is leading to develop new high-power converter topologies to drive different types of high-power systems in the multi-megawatt range. In these systems, the power losses have to be kept below acceptable limits leading to reduce the switching losses as much as possible. In this way, a limited switching frequency (a few hundreds of hertzs) is applied to the power converters generating output waveforms with high distortion. In order to reduce the harmonic distortion content, the first solution is to choose an appropriate modulation technique.

The pulse width modulation (PWM) methods are based on the generation of the switching pattern of the power converter comparing the reference voltages with triangular carriers of frequency $f_{c}$. The carrier frequency $f_{c}$ has to be low in order to keep the switching losses below acceptable limits. The obtained switching pattern generates the desired fundamental frequency but presents high distortion around $f_{c}$ component. In this way, the distortion in the $f_{c}$ component is located in the low frequency range making expensive the filtering stage. Summarizing, the conventional PWM, a well-known solution for medium-power applications, cannot be used for high-power systems.

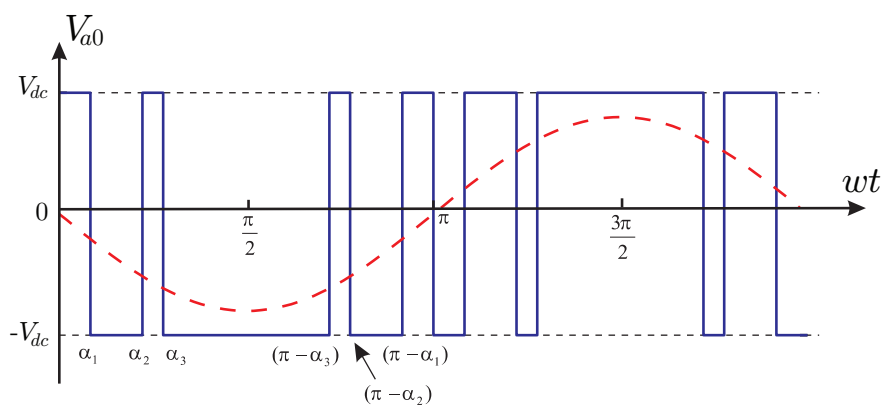

Fig. 1. Voltage waveform with three switching angles to be used in the conventional pre-programmed PWM techniques.

In order to solve this problem, several pre-programmed PWM techniques have been introduced. These strategies are based on the offline calculation of voltage waveforms which have the desired harmonic spectrum. The voltage waveforms are defined using a limited number of switching angles $\alpha_{j}$ $(j=0, \ldots, k-1)$ in the first quarter of period. An example of this pre-defined voltage waveform with three switching angles is represented in Fig. 1.

The selective harmonic elimination technique (SHE) sets the amplitude of the fundamental harmonic and makes zero $k-1$ harmonics where $k$ is the number of switching angles used per quarter of period [1]. Usually, the low order harmonics are chosen to be eliminated reducing the cost (economical cost, size and weight) of the necessary filters. Recently an alternative pre-programmed PWM method has been presented. The selective harmonic mitigation technique (SHM) [2], [3] uses also the pre-defined waveforms represented in Fig. 1 but it does not try to eliminate some harmonics. The SHM technique sets the amplitude of the fundamental harmonic and keeps the harmonic distortion below the limits imposed by a grid code, such as for instance the EN 50160 [4] and CIGRE WG 36-05 [5]). In Table I, all the limits specified in these specific grid codes are detailed.

On the other hand, another strategy to reduce the harmonic distortion keeping limited the number of switchings is to use a multilevel converter topology. The multilevel converters can 
TABLE I

GRID CODE EN 50160 REQUIREMENTS + QUALITY GRID CODE CIGRE WG 36-05

\begin{tabular}{|c|c|c|c|c|c|}
\hline \multicolumn{2}{|c|}{ Odd non-triplen harmonics } & \multicolumn{2}{|c|}{ Odd triplen harmonics } & \multicolumn{2}{|c|}{ Even Harmonics } \\
\hline $\begin{array}{l}\text { Harmonic } \\
\text { order }(n)\end{array}$ & $\begin{array}{c}\text { Relative } \\
\text { Voltage }\left(L_{i}\right)\end{array}$ & $\begin{array}{l}\text { Harmonic } \\
\text { order }(n)\end{array}$ & $\begin{array}{c}\text { Relative } \\
\text { Voltage }\left(L_{i}\right)\end{array}$ & $\begin{array}{l}\text { Harmonic } \\
\text { order }(n)\end{array}$ & $\begin{array}{c}\text { Relative } \\
\text { Voltage }\left(L_{i}\right)\end{array}$ \\
\hline 5 & $6 \%$ & 3 & $5 \%$ & 2 & $2 \%$ \\
\hline 7 & $5 \%$ & 9 & $1.5 \%$ & 4 & $1 \%$ \\
\hline 11 & $3.5 \%$ & 15 & $0.5 \%$ & $6 \ldots 10$ & $0.5 \%$ \\
\hline 13 & $3 \%$ & 21 & $0.5 \%$ & $>10$ & $0.2 \%$ \\
\hline 17 & $2 \%$ & $>21$ & $0.2 \%$ & & \\
\hline 19 & $1.5 \%$ & & & & \\
\hline 23 & $1.5 \%$ & & & & \\
\hline 25 & $1.5 \%$ & & & & \\
\hline$>25$ & $0.2+32.5 / n$ & & & & \\
\hline
\end{tabular}

generate output waveforms with a high number of voltage levels at the expense of using a more complex topology. The pre-programmed SHE and SHM techniques have been applied successfully to multilevel converters.

This paper is organized as follows; in section II the main characteristics of the pre-programmed PWM techniques are introduced. Secondly, section III describes the MPC principle. The proposed MPC based SHM technique is presented in section IV. The obtained results using the MPC-SHM method are presented in section V. Finally, the conclusions of the paper are detailed in section VI.

\section{PRE-PROGRAMMED PWM TECHNIQUES}

As has been introduced previously, a pre-defined voltage waveform with $k$ switching angles $\alpha_{j}(j=0, \ldots, k-1)$ has a harmonic spectrum that can be studied using the Fourier analysis. The amplitude of each harmonic can be obtained using the following expression where $H_{i}$ is the amplitude of the $i^{\text {th }}$ harmonic:

$$
H_{i}=\frac{4 V_{d c}}{i \pi} \sum_{j=0}^{k-1}\left[(-1)^{j} \sin \left(i \alpha_{j}\right)\right] .
$$

\section{A. Selective Harmonic Elimination Technique (SHE)}

The SHE technique sets the amplitude of the $1^{\text {st }}$ order harmonic to a desired value, called modulation index $\left(M_{a}\right)$, and makes zero the amplitude of a limited number of harmonics. Using $k$ switching angles, a maximum of $k-1$ harmonics can be eliminated. Normally, the lowest odd non-triplen harmonics $(5,7,11, \ldots)$ are the most interesting harmonics to be eliminated. This is because, due the symmetry of the waveform, even harmonics have zero amplitude and triplen harmonics can not appear in topologies without neutral connection. The SHE technique is based on solving the following system of equations where $i=5,7,11, \ldots, q$ and $q$ is the highest harmonic order to be zeroed:

$$
\begin{aligned}
H_{1} & =\frac{4 V_{d c}}{\pi} \sum_{j=0}^{k-1}\left[(-1)^{j} \sin \left(\alpha_{j}\right)\right], \\
0 & =\frac{4 V_{d c}}{i \pi} \sum_{j=0}^{k-1}\left[(-1)^{j} \sin \left(i \alpha_{j}\right)\right] .
\end{aligned}
$$

Solving these equations, the values of the switching angles are determined for each value of the modulation index. Usually, these results can be stored in a look up table (LUT) where the input variable is the modulation index and the output are the corresponding switching angles [1].

\section{B. Selective Harmonic Mitigation Technique (SHM)}

The SHM technique takes into account the actual requirements of the power quality summarized in standards and grid codes. These requirements do not force to eliminate some specific harmonics but to reduce them below some acceptable limits. Taking into account this fact, the SHE technique cannot meet these grid codes because the non-eliminated harmonics are not controlled and their values can be above the limits imposed by the grid codes. In addition, the elimination of all the harmonics taken into account in the regulations using the SHE technique would lead to use a high number of switching angles and, consequently, to increase the switching frequency and the corresponding switching losses to unacceptable values.

The SHM technique can be formulated with the following system of inequalities where $M_{a}$ is the modulation index and $L_{i}$ is the maximum limit of harmonic $i^{t h}(i=5,7,11, \ldots, 49)$ imposed by the applied grid code:

$$
\begin{aligned}
& D_{1}=\left|M_{a}-H_{1}\right| \leq L_{1}, \\
& D_{i}=\frac{1}{\left|H_{1}\right|} \frac{4 V_{d c}}{i \pi} \sum_{j=0}^{k-1}\left[(-1)^{j} \sin \left(i \alpha_{j}\right)\right] \leq L_{i} .
\end{aligned}
$$

In previous publications, these equations have be arranged into an cost function to be minimized by an optimization method. This function could be defined as follows: 


$$
O F=\sum_{i=1,5, \ldots, 49} c_{i}\left(D_{i}-L_{i}\right)^{2}+c_{T H D} T H D .
$$

Several optimization methods as tabu search, ant colony, particle swarm or simulated annealing can be used to solve the system. Using any of these optimization algorithms, the values of the switching angles are determined for each value of the modulation index building the corresponding LUT.

\section{SHE versus SHM}

In previous publications, it has been demonstrated that the SHM technique is able to generate output waveforms with better harmonic performance compared to the SHE method. If a high enough number of switching angles is used (15 switching angles in [2]), the SHM technique can fulfill the grid code without any extra filtering stage. This cannot be achieved by the SHE method because some harmonics have unacceptable limits because they are not controlled. If the number of switching angles has to be reduced (usual condition in high-power systems), the SHM technique also achieves better results than the SHE method as has been shown in [3]. However, in this case, as the switching frequency is very limited, although both SHE and SHM methods cannot meet the grid codes, the SHM technique is able to reduce the filters needed to fulfill the power quality requirements.

However, it is important to notice that, both SHE and SHM methods, are based on offline calculations and assume that the modulation index is constant during at least one period (20 milliseconds for a $50 \mathrm{~Hz}$ waveform). This fact makes that these pre-programmed PWM techniques have a low dynamic performance reducing the number of potential applications of these modulation techniques. In fact, this is an important drawback of SHE and SHM and several recent publications have tried to improve their dynamic performance with limited success [6].

Recently, a SHE technique based on the model predictive control (MPC) has been presented [7]. This SHE-MPC method is an online technique that takes advantage of the discrete model of the power converter and the sliding discrete fourier transform (SDFT) to determine the harmonic distortion of the output voltage waveforms.

\section{Basic Operation of the Model Predictive CONTROL}

Any power converter is formed by the connection of different power devices and its operation is defined by the switching of the semiconductors. In this way, a power converter has a finite number of states defining the possible switching of the converter. For instance, if a conventional three-phase two-level voltage source converter is used, only eight possible switching states can be applied to the converter.

The MPC technique is based on the prediction of the control variables using an accurate model of the power system [8]. A cost function $g$ is defined where the control targets are described as error factors which have to be minimized. When a sample of the system is taken, during the sampling time $T_{s}$ the MPC controller predicts the behavior of the system for all the possible switching states of the converter. Each possible switching state of the converter is evaluated determining the corresponding values of the cost function. Finally, the MPC technique applies during the next $T_{s}$ the switching state that minimized the cost function.

In [9], several cost functions are introduced to control different targets such as the currents, the torque and flux, the common-mode voltage, the dc voltage balancing and the reactive power compensation, among others. The cost function can be simple only containing control variables with the same nature (for instance, the output currents) but it can include several terms of different nature. As the different terms could have different units, it is necessary to introduce weighting factors $\lambda_{i}$ to obtain good results from the MPC technique. In this way, the cost function could be defined as the sum of different control objectives, each one with the corresponding weighting factor as follows:

$$
g=\left|x_{1}^{*}-x_{1}^{p}\right|+\sum_{i=1}^{n-1} \lambda_{i}\left|x_{i+1}^{*}-x_{i+1}^{p}\right|,
$$

where the variables to be controlled are denoted as $x_{i}(t)$ $(i=1, \ldots, n)$ and their corresponding predicted values are $x_{i}^{p}(t)$.

The tuning procedure of these weighting factors is not obvious and is one of the drawbacks of the MPC technique. However, recently a simple procedure was introduced to tune the weighting factors [10].

\section{Proposed MPC based SHM technique}

\section{A. Model of the converter}

The proposed MPC-SHM technique is applied to a threephase multilevel cascaded converter and it is convenient to consider the three phases simultaneously using the space vector representation in the $\alpha \beta$ frame. As in the MPC-SHE case introduced in [7], to reduce the number of calculations, and avoid larger $\mathrm{dv} / \mathrm{dts}$, only the six closest vectors and the present space vector (seven in total) are considered for the prediction.

\section{B. Cost function}

The control objective of the MPC-SHM is to follow the voltage reference, to control the low order harmonic distortion and to reduce the switching losses as much as possible. These three objectives are included in the following cost function

$$
\begin{aligned}
g & =S D F T_{f 0}\left\{\left|\mathbf{v}_{s}^{*}-\mathbf{v}_{s}^{p}\right|\right\} \\
& +S D F T_{f 1}\left\{\left|\mathbf{v}_{s}^{*}-\mathbf{v}_{s}^{p}\right|\right\} \\
& +\sum_{i=2}^{N} \lambda_{i} S D F T_{f i}\left\{\left|\mathbf{v}_{s}^{*}-\mathbf{v}_{s}^{p}\right|\right\} \\
& +\lambda_{c} x_{s w}^{p} .
\end{aligned}
$$


In this cost function, the sliding discrete fourier transform (SDFT) is used. The SDFT is a recursive implementation of the discrete Fourier Transform algorithm used to calculate a finite number of single frequency spectral components with very low computational cost [11], [12].

The first and second terms of the cost function, (6) and (7), evaluate the error between the dc component and the fundamental harmonic of the reference and the predicted output voltage respectively.

The third term (8) is the sum of the distortion of those harmonics that have to be kept below acceptable limits imposed by the grid code from the $2^{n d}$ harmonic to the $\mathrm{N}^{t h}$. Taking into account this third term of the cost function, the switching state that minimizes the distortion of these undesired harmonics will be chosen. The weighting factors $\lambda_{i}$ are used to control the importance of each one of the harmonics in the cost function. In this way, the effort trying to reduce the harmonic distortion of harmonic order $i^{t h}$ can be relaxed or strengthened depending on designing considerations. This fact will be very useful for the final proposed control design.

Finally, the fourth term (9) is used to reduce the number of commutations. This term is necessary since the first and second terms could force a high number of commutations in order to achieve the control targets. The variable $x_{s w}^{p}$ is the predicted number of commutations that will be performed when changing from the present output voltage vector $\mathbf{v}_{s}[k]$ to the predicted vector $\mathbf{v}_{s}^{p}[k+1]$. Here the weighting factor $\lambda_{s w}$ is used to tune the weight of this term within the cost function.

\section{Control design}

The cost function previously defined can be used to achieve the SHE control target, that is to eliminate the distortion of harmonics from $2^{\text {nd }}$ up to $\mathrm{N}^{t h}$. The idea introduced in this paper is to use variable weighting factors $\lambda_{i}$ relaxing the condition of eliminating the distortion of those harmonics. The proposed MPC-SHM technique can be summarized using the block diagram represented in Fig. 2 where a weighting factors tuning block has been added to the previously introduced MPC-SHE method [7]. This additional block is only executed each period of the reference voltage that is $20 \mathrm{~ms}$ while the MPC blocks are executed each sampling time $T_{s}$.

In the proposed MPC-SHM technique, the weighting factors are defined as

$$
\lambda_{i}= \begin{cases}\lambda_{0 i}, & \text { for } i=0,1 \\ \sigma_{i} \lambda_{0 i}, & \text { for } i=2, \ldots, N\end{cases}
$$

where $\lambda_{0 i}$ is the initial value of the weighting factor corresponding to $i^{t h}$ harmonic. The factors $\lambda_{i}$ are modified by $\sigma_{i}$ taking into account the normalized errors of the harmonic distortion of $i^{\text {th }}$ harmonic defined as

$$
E_{i}=D_{i}-m_{1} L_{i}
$$

where $D_{i}$ was defined in (3), $L_{i}$ is the maximum distortion imposed by the grid code for $i^{\text {th }}$ harmonic and factor $m_{1}$ is

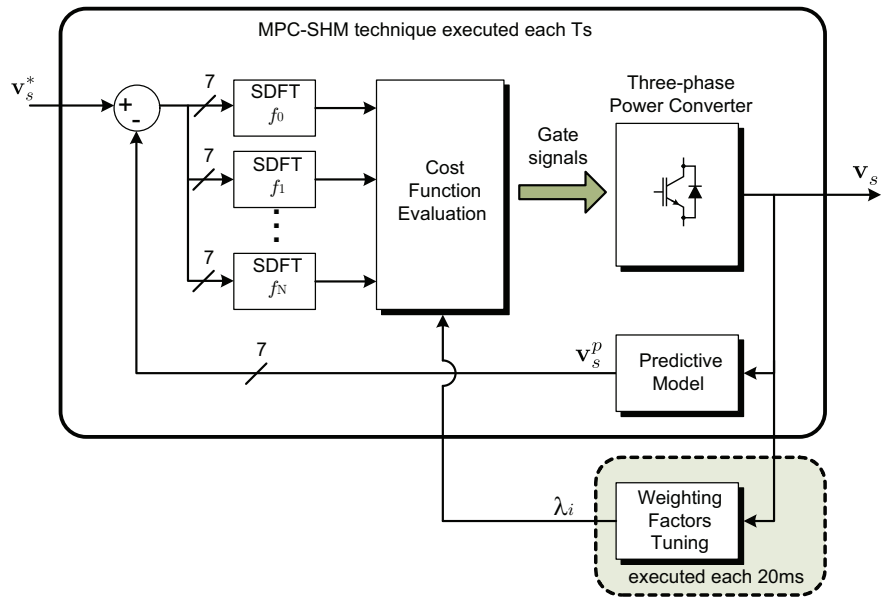

Fig. 2. Block diagram of the proposed MPC-SHM technique.

TABLE II

INITIAL VALUES OF THE WEIGHTING FACTORS $\lambda_{0 i}$ FOR HARMONIC $i^{t h}$

\begin{tabular}{c|c|c|c|c|c|c|c|c}
\hline \hline$\lambda_{02}$ & $\lambda_{03}$ & $\lambda_{04}$ & $\lambda_{05}$ & $\lambda_{06}$ & $\lambda_{07}$ & $\lambda_{08}$ & $\lambda_{09}$ & $\lambda_{010}$ \\
\hline 0.07 & 0.003 & 0.5 & $9 \cdot 10^{-5}$ & 0.6 & 0.007 & 1 & 0.1 & 0.8 \\
\hline \hline
\end{tabular}

introduced to create a margin of safety between the obtained distortion and the limit imposed by the grid code. For instance, if $m_{1}$ is equal to 0.8 , it means that factor $\sigma_{i}$ will be higher than 1 if the normalized distortion of harmonic $i^{t h}$ is higher than $80 \%$ of the limit imposed by the grid code.

The factors $\sigma_{i}$ are defined as a piecewise function that depends on the normalized distortion error $E_{i}$ as follows

$$
\sigma_{i}= \begin{cases}1+k p_{i} E_{i}, & \text { if } D_{i}>m_{1} L_{i} \\ 1-k p_{i} E_{i}, & \text { if } D_{i}<m_{2} L_{i} \\ 1, & \text { otherwise }\end{cases}
$$

where $k p_{i}$ is defined as a proportional gain and $m_{2}$ marks the margin where the weighting factors are lower than 1 . For instance, if $m_{2}$ is equal to 0.5 , it means that factor $\sigma_{i}$ will be lower than 1 if the normalized distortion of harmonic $i^{\text {th }}$ is lower than $50 \%$ of the limit imposed by the grid code.

\section{Simulation Results}

Several three-level power cells formed by full H-bridges can be associated to build a converter with a higher number of levels. In general, if $m$ power cells are connected in series to build the converter and all the cells have the same dc voltage, the number of levels that can be achieved is $2 m+1$. This topology is named symmetrical $m$-cell $\mathrm{CHB}$ converter and it presents great properties as a high modularity and reduced number of switches [13], [14].

This section presents the results obtained using the MPCSHM technique in a seven-level three-cell symmetrical CHB converter. In the computing process, the limits specified in the EN 50160 [4] and CIGRE WG 36-05 [5] grid codes have been considered but any other could have been chosen. 

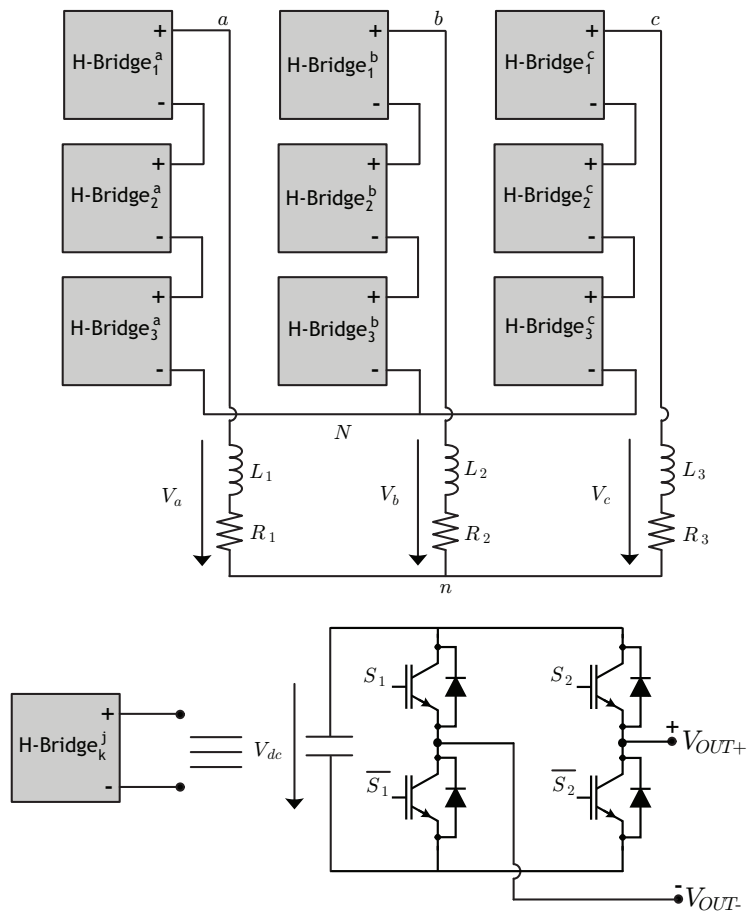

Fig. 3. Three-phase symmetrical three-cell cascaded converter based on the series connection of three H-bridge power cells.

Results for a three-phase three-cell seven-level multilevel cascaded converter shown in Fig. 3 were obtained for a resistive inductive load $(\mathrm{L}=17 \mathrm{mH}, \mathrm{R}=20 \Omega)$. The dc-link voltage of each cell of the power converter is $V_{d c}=66.6 \mathrm{~V}$, giving a maximum amplitude of $200 \mathrm{~V}$. The fundamental frequency of the reference voltage is $50 \mathrm{~Hz}$, and the harmonics taken into account in the MPC-SHM technique are: 0, 2, 3, 4, 5, 6, $7,8,9$ and 10 . The sample period to perform the predictive algorithm is $T_{s}=50 \mu \mathrm{s}$, using two step ahead predictions. The initial values of the weighting factors, obtained offline by iterative simulations, are summarized in Table II while $\lambda_{00}$ and $\lambda_{01}$ are equal to 0.3 and 1 respectively. Factors $k p_{i}$ are equal to 250 except $k p_{5}$ that is equal to 5000. Factors $m_{1}$ and $m_{2}$ are defined as 0.8 and 0.5 respectively.

Figure 4 shows the converter phase output voltage $V_{a N}$, the line-line voltage $V_{a b}$, the load voltage $V_{a n}$ and the load currents $i_{a}, i_{b}$ and $i_{c}$. In addition the respective harmonic spectra are shown next to each waveform. These results are summarized in Table III where the information about the obtained harmonic distortion in the line voltage $V_{a b}$ is included. It can be clearly observed that the proposed method effectively reduces the distortion of all the harmonics taken into account. In addition the fundamental component is accurately controlled and load currents are highly sinusoidal. It can also be appreciated in the phase voltage that the algorithm performs only a few commutations per cycle. This can be clearly observed in Fig. 5, where the transient of the average switching frequency is plotted. The final average switching frequency over one second of operation is equal to $127.7 \mathrm{~Hz}$ using the MPC-SHM technique.
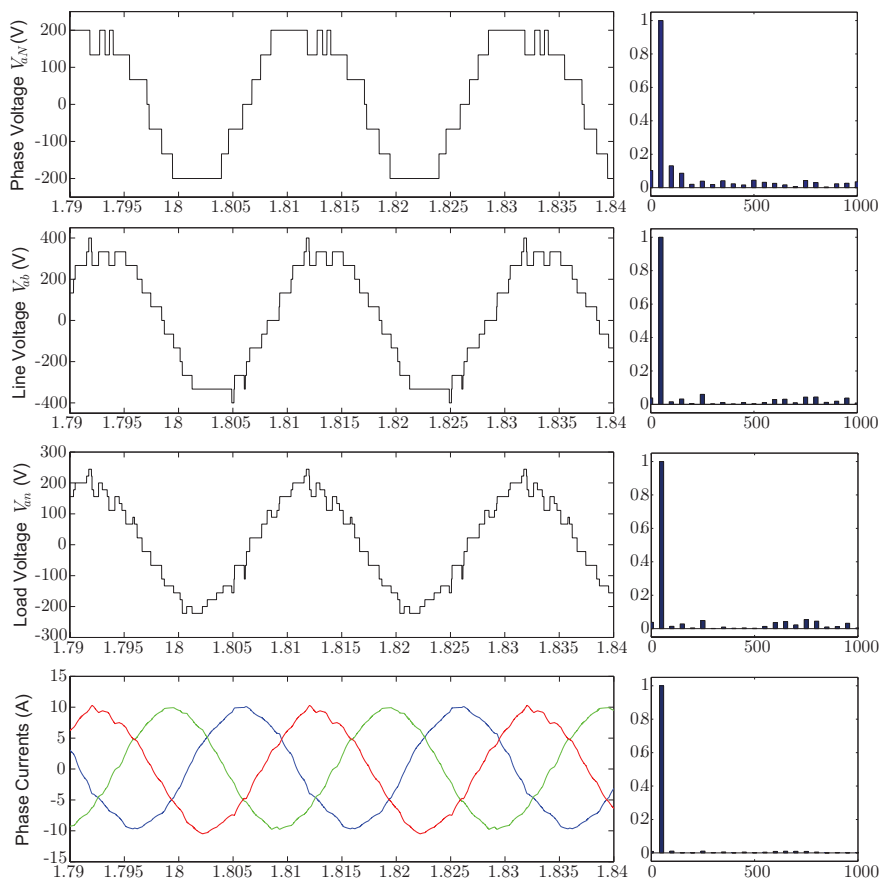

Fig. 4. Predictive harmonic mitigation phase output voltage $V_{a N}$, line voltage $V_{a b}$, load voltage $V_{a n}$ and load currents $i_{a}, i_{b}, i_{c}$ for a 7-level CHB.

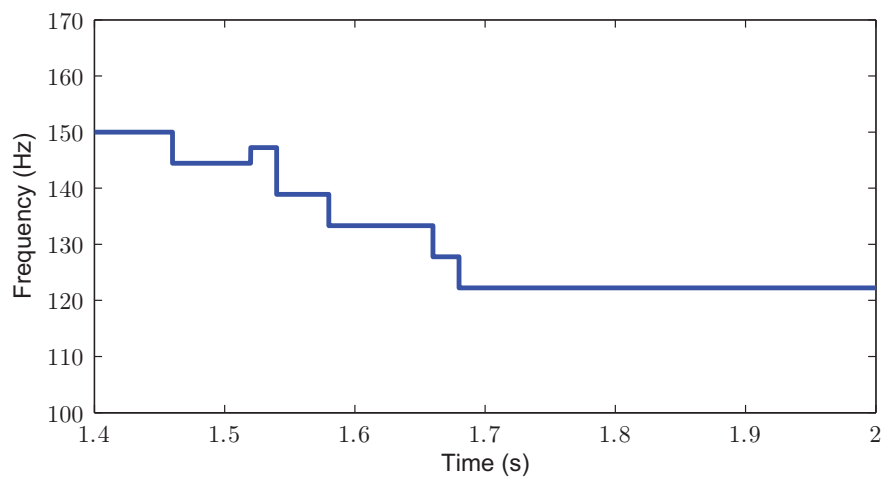

Fig. 5. Average switching frequency obtained by the MPC-SHM technique with a modulation index in the reference voltage equal to 1 .

TABLE III

HARMONIC DISTORTION OBTAINED WITH THE MPC-SHM WITH MODULATION INDEX EQUAL TO 1

\begin{tabular}{c|c|c}
\hline \hline Harmonic order & Grid Code limit (\%) & MPC-SHM result (\%) \\
\hline 2 & 2 & 1.58 \\
3 & 5 & 1.66 \\
4 & 1 & 0.35 \\
5 & 6 & 3.12 \\
6 & 0.5 & 0.32 \\
7 & 5 & 2.01 \\
8 & 0.5 & 0.12 \\
9 & 1.5 & 1.09 \\
10 & 0.5 & 0.2 \\
\hline \hline
\end{tabular}

The same experiment has been carried out changing the 
TABLE IV

WORST HARMONIC DISTORTION OBTAINED WITH THE MPC-SHM WITH MODULATION INDEX FROM 0.6 TO 1.25

\begin{tabular}{c|c|c}
\hline \hline Harmonic order & Grid Code limit (\%) & MPC-SHM result (\%) \\
\hline 2 & 2 & 1.98 \\
3 & 5 & 4.79 \\
4 & 1 & 0.65 \\
5 & 6 & 5.97 \\
6 & 0.5 & 0.42 \\
7 & 5 & 2.96 \\
8 & 0.5 & 0.36 \\
9 & 1.5 & 1.49 \\
10 & 0.5 & 0.33 \\
\hline \hline
\end{tabular}

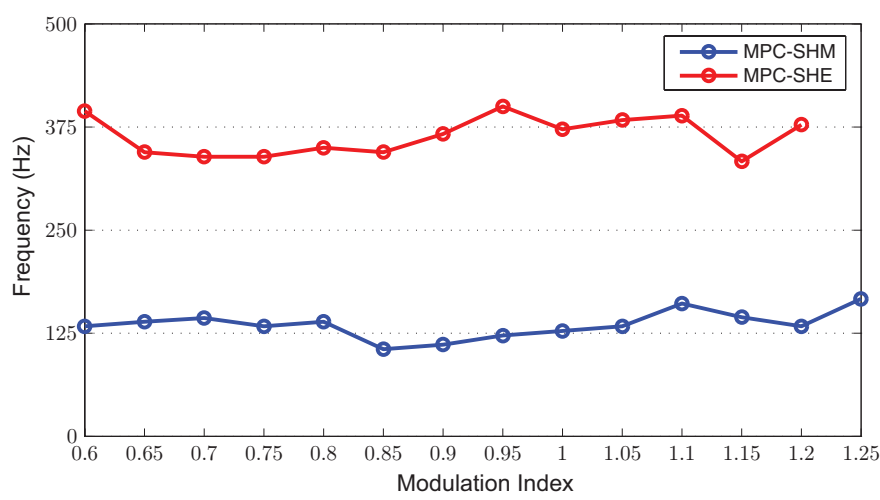

Fig. 6. MPC-SHM average switching frequency compared with the results obtained using the MPC-SHE [7] for the three-cell seven-level CHB.

modulation index value from 0.6 to 1.25 obtaining a high performance. In Table IV, the worst harmonic distortion for each harmonic taken into account in the MPC-SHM technique for the complete modulation index range have been summarized. It can be observed that even in the worst case, the distortion is always below the limit imposed by the grid code. In addition, in Fig. 6 the average switching frequency for each modulation index value has been represented. It can be observed that the power losses will be kept very low independently of the modulation index value. In addition, the results have been compared with the corresponding ones using the MPC-SHE technique. It can be observed that the harmonic mitigation concept helps to drastically decrease the average switching frequency of the converter.

\section{CONCLUSiOnS}

A modulation method based on model predictive control and using the SDFT algorithm has been presented achieving, with very low switching frequency, a reduced distortion in the low order harmonics. The obtained results are similar to the previously introduced selective harmonic mitigation method. However, the proposed technique is computed online leading to an improvement in the dynamic performance. The method can be applied to any converter topology with any number of levels in a simple way. The obtained results demonstrate that a large modulation index can be used achieving a high performance even in the overmodulation range.

\section{ACKNOWLEDGMENT}

The authors gratefully acknowledge the financial support provided by the Ministry of Education under grant PR20100162, research project TEC2007-61879 and project TEC201017782 and by Fondecyt 1110783 and CCTVal ( ${ }^{\circ}$ FB0821).

\section{REFERENCES}

[1] H. S. Patel and R. G. Hoft, "Generalized techniques of harmonic elimination and voltage control in thyristor inverters-Part 1: Harmonic elimination," IEEE Trans. Ind. Applicat., vol. IA-9, pp. 310-317, May/June 1973.

[2] L. G. Franquelo, J. Napoles, R. Portillo, J. I. Leon and M. A. Aguirre, "A Flexible Selective Harmonic Mitigation Technique to Meet Grid Codes in Three-Level PWM Converters," IEEE Trans. Ind. Electron., vol. 54, no. 6 , pp. 3022-3029, Dec. 2007.

[3] J. Napoles, J. I. Leon, R. Portillo, L. G. Franquelo and M. A. Aguirre, "Selective Harmonic Mitigation Technique for High-Power Converters," IEEE Trans. Ind. Electron., vol. 57, no. 7, pp. 2315-2323, July 2010.

[4] CENELEC EN 50160, "Voltage characteristics of electricity supplied by public distribution systems," 2001.

[5] CIGRE WG 36-05, "Harmonics, characteristic parameters, methods of study, estimates of existing values in the network," Electra no.77, 1981, S.35-54.

[6] J. Nápoles, R. Portillo, J. I. Leon, M. A. Aguirre and L. G. Franquelo, "Implementation of a closed loop SHMPWM Technique for Three Level Converters," in the $34^{\text {th }}$ IEEE Annual Conference of the Industrial Electronics Society (IECON'08), pp. 3260-3265, 10-13 Nov. 2008, Orlando (USA).

[7] S. Kouro, B. La Rocca, S. Alepuz, B. Wu and J. Rodriguez, "Predictive Control Based Selective Harmonic Elimination With Low Switching Frequency for Multilevel Converters," IEEE Energy Conversion Congress and Exposition, ECCE'09, pp. 3130-3136, San Jose (United States), 20-24 Sept. 2009.

[8] J. M. Quero, E. F. Camacho and L. G. Franquelo, "Neural network for constrained predictive control," IEEE Transactions on Circuits and Systems I: Fundamental Theory and Applications, vol. 40, no. 9, pp. 621-2626, Sept. 1993.

[9] S. Kouro, P. Cortes, R. Vargas, U. Ammann and J. Rodriguez, "Model Predictive Control - A Simple and Powerful Method to Control Power Converters," IEEE Trans. Ind. Electron., vol. 56, no. 6, pp. 1826-1838, June 2009.

[10] P. Cortes, S. Kouro, B. La Rocca, R. Vargas, J. Rodriguez, J. I. Leon, S Vazquez and L. G. Franquelo, "Guidelines for weighting factors design in Model Predictive Control of power converters and drives," IEEE International Conference on Industrial Technology ICIT'09, pp. 1-7, Melbourne (Australia), 10-13 Feb. 2009.

[11] E. Jacobsen and R. Lyons, "The Sliding DFT," IEEE Signal Processing Mag., vol. 20, no. 2, pp. 74-80, Mar. 2003.

[12] E. Jacobsen and R. Lyons, "An Update to the Sliding DFT," IEEE Signal Processing Mag., vol. 21, no. 1, pp. 110-111, Jan. 2004

[13] J. Rodriguez, L. G. Franquelo, S. Kouro, J. I. Leon, R. Portillo, M. M. Prats and M. Perez, "Multilevel Converters: An Enabling Technology for High-Power Applications," Proceedings of the IEEE, vol. 97, no. 11, pp. 1786-1817, Nov. 2009.

[14] S. Kouro, M. Malinowski, K. Gopakumar, J. Pou, L. G. Franquelo, B. Wu, J. Rodriguez, M. Perez and J. I. Leon, "Recent Advances and Industrial Applications of Multilevel Converters," IEEE Trans. Ind. Electron., vol. 57, no. 8, pp. 2553-2580, Aug. 2010. 